\title{
Differential Harnack Estimates for a Semilinear Parabolic System
}

\author{
Hui Wu \\ School of Mathematical Sciences, Qufu Normal University, Qufu 273165, China \\ Correspondence should be addressed to Hui Wu; wuhui8668@126.com
}

Received 4 August 2018; Accepted 15 January 2019; Published 10 March 2019

Academic Editor: Mitsuru Sugimoto

Copyright (C) 2019 Hui Wu. This is an open access article distributed under the Creative Commons Attribution License, which permits unrestricted use, distribution, and reproduction in any medium, provided the original work is properly cited.

In this paper, we prove differential Harnack inequalities for positive solutions of a semilinear parabolic system on hyperbolic space. We use the inequalities to construct classical Harnack estimates by integrating along space-time.

\section{Introduction}

In this paper, we study the following problem:

$$
\begin{aligned}
f_{t} & =\Delta f+\mathrm{e}^{\mu t} g^{p}, \quad \mathbb{R}^{n} \times(0,+\infty), \\
g_{t} & =\Delta g+\mathrm{e}^{\nu t} f^{q}, \quad \mathbb{R}^{n} \times(0,+\infty), \\
f(x, 0) & =f_{0}, \\
g(x, 0) & =g_{0},
\end{aligned}
$$

$$
\mathbb{R}^{n}
$$

where $p, q, \mu, \nu$ are positive constants.

P. Li and S.-T.Yau in [1] were first pioneers to the study of differential Harnack inequalities which were brought to general parabolic geometric flows by R. Hamilton (see [2]). Using these inequalities can derive ancient solutions, bounds on gradient Ricci solitons, Holder continuity. Differential Harnack inequalities are important aspects of properties of partial differential equations. Paper [3] described differential Harnack inequalities to the initial value problem of a semilinear parabolic equation when the semilinear term is $e^{\mu t} f^{p}, \mu>0$. There have been numerous interesting results on the properties of solutions of partial differential equations, such as existence of solutions [4-17], nonexistence and blowup of solutions [18-22], and asymptotic behaviors of solutions [23-28].

Let $(f(x, t), g(x, t))$ be positive smooth solutions to (1) and $(u, v):=(\log f, \log g)$. The main object of our study is the following Harnack quantities:

$$
\begin{aligned}
& H_{1} \equiv \alpha \Delta u+\beta_{1}|\nabla u|^{2}+c e^{\mu t+p v-u}+\psi_{1}(t)+\phi_{1}(x), \\
& H_{2} \equiv \alpha \Delta v+\beta_{2}|\nabla v|^{2}+c e^{v t+q u-v}+\psi_{2}(t)+\phi_{2}(x),
\end{aligned}
$$

where $\alpha, \beta_{i}, c \in \mathbb{R}, \alpha>\max \left\{\beta_{1}, \beta_{2}\right\}$ and $\psi_{i}, \phi_{i}$ will be chosen suitably $i=1,2$.

We will derive our differential Harnack estimate.

Theorem 1. Let $(f(x, t), g(x, t))$ be positive classical solutions to (1), and $(u, v):=(\log f, \log g)$. If $\alpha, \beta_{i}, k_{i}$, and c satisfy

$$
\begin{aligned}
& \alpha>\max \left\{\beta_{1}, \beta_{2}\right\} \geq 0 \\
& k_{1}-p k_{2} \geq 0 \\
& k_{2}-q k_{1} \geq 0 \\
& k_{i} \geq \frac{n \alpha^{2}}{2\left(\alpha-\beta_{i}\right)}>0 \\
& -\beta_{2}+\alpha p+c(1-p) \geq 0 \\
& -\beta_{1}+\alpha q+c(1-q) \geq 0 \\
& \frac{4}{n \alpha^{2}} \beta_{1} c+1-\frac{p^{2}\left(\alpha-\beta_{1}\right)}{-p \beta_{2}+\alpha p^{2}+c p(1-p)} \geq 0 \\
& \frac{4}{n \alpha^{2}} \beta_{2} c+1-\frac{q^{2}\left(\alpha-\beta_{2}\right)}{-q \beta_{1}+\alpha q^{2}+c q(1-q)} \geq 0
\end{aligned}
$$


then we have

$$
\begin{aligned}
& H_{1} \equiv \alpha \Delta u+\beta_{1}|\nabla u|^{2}+c \mathrm{e}^{\mu t+p v-u}+\frac{k_{1}}{t} \geq 0, \\
& H_{2} \equiv \alpha \Delta v+\beta_{2}|\nabla v|^{2}+c \mathrm{e}^{\mu t+q u-v}+\frac{k_{2}}{t} \geq 0,
\end{aligned}
$$

for all $t$.

The paper is organized as follows. In Section 2 we prove Theorem 1 which describes differential Harnack estimate. There are applications of Theorem 1 in Section 3.

\section{Harnack Estimate}

In this section, we shall first obtain our differential Harnack inequalities, relying on the parabolic maximum principle.

Lemma 2. Suppose $(f(x, t), g(x, t))$ are positive solutions to (1) and $(u, v):=(\log f, \log g)$ and $\left(H_{1}, H_{2}\right)$ are defined as in (2). Assume that $\alpha, \beta_{1}, \beta_{2}$, and c satisfy

$$
\begin{aligned}
& \alpha>\max \left\{\beta_{1}, \beta_{2}\right\} \geq 0, \\
& -\beta_{2}+\alpha p+c(1-p) \geq 0, \\
& -\beta_{1}+\alpha q+c(1-q) \geq 0, \\
& \frac{4}{n \alpha^{2}} \beta_{1} c+1-\frac{p^{2}\left(\alpha-\beta_{1}\right)}{-p \beta_{2}+\alpha p^{2}+c p(1-p)} \geq 0, \\
& \frac{4}{n \alpha^{2}} \beta_{2} c+1-\frac{q^{2}\left(\alpha-\beta_{2}\right)}{-q \beta_{1}+\alpha q^{2}+c q(1-q)} \geq 0 .
\end{aligned}
$$

Then we have

$$
\begin{aligned}
\partial_{t} H_{1} \geq & \Delta H_{1}+2 \nabla H_{1} \cdot \nabla u+h_{11} H_{1} \\
& +e^{\mu t+p v-u}\left(p H_{2}-H_{1}\right)+h_{12} \\
\partial_{t} H_{2} \geq & \Delta H_{2}+2 \nabla H_{2} \cdot \nabla v+h_{21} H_{2} \\
& +\mathrm{e}^{v t+q u-v}\left(q H_{1}-H_{2}\right)+h_{22},
\end{aligned}
$$

where

$$
\begin{aligned}
h_{11} & =2\left(\alpha-\beta_{1}\right) \\
& \cdot \frac{1}{n \alpha^{2}}\left\{H_{1}-2\left(\beta_{1}|\nabla u|^{2}+c \mathrm{e}^{\mu t+p v-u}+\psi_{1}+\phi_{1}\right)\right\}, \\
h_{12} & =\partial_{t} \psi_{1}-\Delta \phi_{1}+\frac{2}{n \alpha^{2}}\left(\alpha-\beta_{1}\right)\left(\psi_{1}+\phi_{1}\right)^{2} \\
& -\frac{n \alpha^{2}\left|\nabla \phi_{1}\right|^{2}}{4 \beta_{1}\left(\alpha-\beta_{1}\right)\left(\psi_{1}+\phi_{1}\right)} \\
& +\mathrm{e}^{\mu t+p v-u}\left(c \mu+\psi_{1}+\phi_{1}-p\left(\psi_{2}+\phi_{2}\right)\right), \\
h_{21} & =2\left(\alpha-\beta_{2}\right) \\
& \cdot \frac{1}{n \alpha^{2}}\left\{H_{2}-2\left(\beta_{2}|\nabla v|^{2}+c \mathrm{e}^{v t+q u-v}+\psi_{2}+\phi_{2}\right)\right\},
\end{aligned}
$$

$$
\begin{aligned}
h_{22} & =\partial_{t} \psi_{2}-\Delta \phi_{2}+\frac{2}{n \alpha^{2}}\left(\alpha-\beta_{2}\right)\left(\psi_{2}+\phi_{2}\right)^{2} \\
& -\frac{n \alpha^{2}\left|\nabla \phi_{2}\right|^{2}}{4 \beta_{2}\left(\alpha-\beta_{2}\right)\left(\psi_{2}+\phi_{2}\right)} \\
& +\mathrm{e}^{v t+q u-v}\left(c \nu+\psi_{2}+\phi_{2}-q\left(\psi_{1}+\phi_{1}\right)\right) .
\end{aligned}
$$

Proof. Substituting $(f, g)=\left(\mathrm{e}^{u}, \mathrm{e}^{v}\right)$ into (1), we have

$$
\begin{aligned}
& u_{t}=\Delta u+|\nabla u|^{2}+\mathrm{e}^{\mu t+p v-u}, \\
& v_{t}=\Delta v+|\nabla v|^{2}+\mathrm{e}^{v t+q u-v} .
\end{aligned}
$$

Using the above equations, we have

$$
\begin{aligned}
\left(\partial_{t}-\Delta\right) \Delta u \\
=2|\nabla \nabla u|^{2}+2 \nabla u \cdot \nabla \Delta u \\
\quad+e^{\mu t+p v-u}\left(p \Delta v-\Delta u+|p \nabla v-\nabla u|^{2}\right), \\
\left(\partial_{t}-\Delta\right)\left(|\nabla u|^{2}\right) \\
=2 \nabla u \cdot \nabla|\nabla u|^{2}-2|\nabla \nabla u|^{2}+2 \mathrm{e}^{\mu t+p v-u} \nabla u \\
\quad \cdot(p \nabla v-\nabla u) .
\end{aligned}
$$

Furthermore, applying (2) and Cauchy-Schwarz inequality $|\nabla \nabla u|^{2} \geq(1 / n)(\Delta u)^{2}$ yields

$$
\begin{aligned}
\partial_{t} & H_{1}-\Delta H_{1}-2 \nabla H_{1} \cdot \nabla u=2\left(\alpha-\beta_{1}\right)|\nabla \nabla u|^{2} \\
& +\mathrm{e}^{\mu t+p v-u}\left(p H_{2}-H_{1}\right) \\
& +\mathrm{e}^{\mu t+p v-u}\left\{\left(\alpha p^{2}-p \beta_{2}+c p(1-p)\right)|\nabla v|^{2}\right. \\
& \left.-2 p\left(\alpha-\beta_{1}\right) \nabla u \cdot \nabla v\right\}+\mathrm{e}^{\mu t+p v-u}\left(\alpha-\beta_{1}\right)|\nabla u|^{2} \\
& +\mathrm{e}^{\mu t+p v-u}\left(c \mu+\psi_{1}+\phi_{1}-p\left(\psi_{2}+\phi_{2}\right)\right)+\partial_{t} \psi_{1} \\
& -\Delta \phi_{1}-2 \nabla u \cdot \nabla \phi_{1} \geq 2\left(\alpha-\beta_{1}\right) \frac{1}{n \alpha^{2}} H_{1}\left\{H_{1}\right. \\
& \left.-2\left(\beta_{1}|\nabla u|^{2}+c \mathrm{e}^{\mu t+p v-u}+\psi_{1}+\phi_{1}\right)\right\}+2\left(\alpha-\beta_{1}\right) \\
& +\frac{1}{n \alpha^{2}} \beta_{1}\left\{\beta_{1}|\nabla u|^{4}+2|\nabla u|^{2}\left(c \mathrm{e}^{\mu t+p v-u}+\psi_{1}+\phi_{1}\right)\right\} \\
& +2\left(\alpha-\beta_{1}\right) \frac{1}{n \alpha^{2}}\left(c \mathrm{e}^{\mu t+p v-u}+\psi_{1}+\phi_{1}\right)^{2} \\
& +\mathrm{e}^{\mu t+p v-u}\left(p H_{2}-H_{1}\right) \\
+ & \mathrm{e}^{\mu t+p v-u}\left\{p\left(\alpha p-\beta_{2}+c(1-p)\right)|\nabla v|^{2}\right. \\
& \left.-2 p\left(\alpha-\beta_{1}\right) \nabla u \cdot \nabla v\right\}+\mathrm{e}^{\mu t+p v-u}\left(\alpha-\beta_{1}\right)|\nabla u|^{2} \\
& -\Delta u \cdot \nabla \phi_{1}
\end{aligned}
$$


If $\alpha p-\beta_{2}+c(1-p) \geq 0$, the above inequality is

$$
\begin{aligned}
\geq 2 & \left(\alpha-\beta_{1}\right) \frac{1}{n \alpha^{2}} \\
\cdot & H_{1}\left\{H_{1}-2\left(\beta_{1}|\nabla u|^{2}+c \mathrm{e}^{\mu t+p v-u}+\psi_{1}+\phi_{1}\right)\right\} \\
+ & \mathrm{e}^{\mu t+p v-u}\left(p H_{2}-H_{1}\right)+\frac{4 \beta_{1}}{n \alpha^{2}}\left(\alpha-\beta_{1}\right)\left(\psi_{1}+\phi_{1}\right) \\
& \cdot|\nabla u|^{2}+2\left(\alpha-\beta_{1}\right) \\
& \cdot \frac{1}{n \alpha^{2}}\left\{\beta_{1}^{2}|\nabla u|^{4}+\left(c \mathrm{e}^{\mu t+p v-u}+\psi_{1}+\phi_{1}\right)^{2}\right\} \\
+ & \left(\frac{4}{n \alpha^{2}} \beta_{1} c+1-\frac{p\left(\alpha-\beta_{1}\right)}{\alpha p-\beta_{2}+c(1-p)}\right)\left(\alpha-\beta_{1}\right) \\
& \cdot \mathrm{e}^{\mu t+p v-u}|\nabla u|^{2} \\
+ & \mathrm{e}^{\mu t+p v-u}\left(c \mu+\psi_{1}+\phi_{1}-p\left(\psi_{2}+\phi_{2}\right)\right)+\partial_{t} \psi_{1} \\
- & \Delta \phi_{1}-2 \nabla u \cdot \nabla \phi_{1}
\end{aligned}
$$

First note the following inequality:

$$
\begin{aligned}
& \frac{4 \beta_{1}}{n \alpha^{2}}\left(\alpha-\beta_{1}\right)\left(\psi_{1}+\phi_{1}\right)|\nabla u|^{2}-2 \nabla u \cdot \nabla \phi_{1} \\
& \geq-\frac{n \alpha^{2}\left|\nabla \phi_{1}\right|^{2}}{4 \beta_{1}\left(\alpha-\beta_{1}\right)\left(\psi_{1}+\phi_{1}\right)} .
\end{aligned}
$$

If $\alpha>\beta_{1}$ and $\left(4 / n \alpha^{2}\right) \beta_{1} c+1-p^{2}\left(\alpha-\beta_{1}\right) /\left(-p \beta_{2}+\alpha p^{2}+c p(1-\right.$ $p)) \geq 0$, the above inequality is

$$
\begin{aligned}
\geq & 2\left(\alpha-\beta_{1}\right) \frac{1}{n \alpha^{2}} \\
& \cdot H_{1}\left\{H_{1}-2\left(\beta_{1}|\nabla u|^{2}+c \mathrm{e}^{\mu t+p v-u}+\psi_{1}+\phi_{1}\right)\right\} \\
& +\mathrm{e}^{\mu t+p v-u}\left(p H_{2}-H_{1}\right)-\frac{n \alpha^{2}\left|\nabla \phi_{1}\right|^{2}}{4 \beta_{1}\left(\alpha-\beta_{1}\right)\left(\psi_{1}+\phi_{1}\right)} \\
& +\mathrm{e}^{\mu t+p v-u}\left(c \mu+\psi_{1}+\phi_{1}-p\left(\psi_{2}+\phi_{2}\right)\right)+\partial_{t} \psi_{1} \\
& -\Delta \phi_{1}+\frac{2}{n \alpha^{2}}\left(\alpha-\beta_{1}\right)\left(\psi_{1}+\phi_{1}\right)^{2} .
\end{aligned}
$$

For $\mathrm{H}_{2}$, we have similar results.

$$
\begin{aligned}
\partial_{t} & H_{2}-\Delta H_{2}-2 \nabla H_{2} \cdot \nabla v \geq 2\left(\alpha-\beta_{2}\right) \frac{1}{n \alpha^{2}} H_{2}\left\{H_{2}\right. \\
& \left.-2\left(\beta_{2}|\nabla v|^{2}+c e^{v t+q u-v}+\psi_{2}+\phi_{2}\right)\right\}+2\left(\alpha-\beta_{2}\right) \\
& \cdot \frac{1}{n \alpha^{2}} \beta_{2}\left\{\beta_{2}|\nabla v|^{4}+2|\nabla v|^{2}\left(c \mathrm{e}^{v t+q u-v}+\psi_{2}+\phi_{2}\right)\right\} \\
& +2\left(\alpha-\beta_{2}\right) \frac{1}{n \alpha^{2}}\left(c \mathrm{e}^{v t+q u-v}+\psi_{2}+\phi_{2}\right)^{2} \\
& +\mathrm{e}^{v t+q u-v}\left(q H_{1}-H_{2}\right) \\
+ & \mathrm{e}^{v t+q u-v}\left\{q\left(-\beta_{1}+\alpha q+c(1-q)\right)|\nabla u|^{2}\right.
\end{aligned}
$$

$$
\begin{aligned}
& \left.-2 q\left(\alpha-\beta_{2}\right) \nabla u \cdot \nabla v\right\}+\mathrm{e}^{v t+q u-v}\left(\alpha-\beta_{2}\right)|\nabla v|^{2} \\
& +\mathrm{e}^{v t+q u-v}\left(c v+\psi_{2}+\phi_{2}-q\left(\psi_{1}+\phi_{1}\right)\right)+\partial_{t} \psi_{2} \\
& -\Delta \phi_{2}-2 \nabla v \cdot \nabla \phi_{2}
\end{aligned}
$$

If $\alpha q-\beta_{1}+c(1-q) \geq 0$, the above inequality is

$$
\begin{aligned}
\geq 2 & \left(\alpha-\beta_{2}\right) \frac{1}{n \alpha^{2}} \\
\cdot & H_{2}\left\{H_{2}-2\left(\beta_{2}|\nabla v|^{2}+c \mathrm{e}^{v t+q u-v}+\psi_{2}+\phi_{2}\right)\right\} \\
+ & \mathrm{e}^{v t+q u-v}\left(q H_{1}-H_{2}\right)+\frac{4 \beta_{2}}{n \alpha^{2}}\left(\alpha-\beta_{2}\right)\left(\psi_{2}+\phi_{2}\right) \\
& \cdot|\nabla v|^{2}+2\left(\alpha-\beta_{2}\right) \\
& \cdot \frac{1}{n \alpha^{2}}\left\{\beta_{2}^{2}|\nabla v|^{4}+\left(c \mathrm{e}^{v t+q u-v}+\psi_{2}+\phi_{2}\right)^{2}\right\} \\
+ & \left(\frac{4}{n \alpha^{2}} \beta_{2} c+1-\frac{q^{2}\left(\alpha-\beta_{2}\right)}{-q \beta_{1}+\alpha q^{2}+c q(1-q)}\right) \\
& \cdot\left(\alpha-\beta_{2}\right) \mathrm{e}^{v t+q u-v}|\nabla v|^{2} \\
+ & \mathrm{e}^{v t+q u-v}\left(c v+\psi_{2}+\phi_{2}-q\left(\psi_{1}+\phi_{1}\right)\right)+\partial_{t} \psi_{2} \\
- & \Delta \phi_{2}-2 \nabla v \cdot \nabla \phi_{2} \cdot
\end{aligned}
$$

If $\left(4 / n \alpha^{2}\right) \beta_{2} c+1-q^{2}\left(\alpha-\beta_{2}\right) /\left(-q \beta_{1}+\alpha q^{2}+c q(1-q)\right) \geq 0$, the above inequality is

$$
\begin{aligned}
\geq & 2\left(\alpha-\beta_{2}\right) \frac{1}{n \alpha^{2}} \\
& \cdot H_{2}\left\{H_{2}-2\left(\beta_{2}|\nabla v|^{2}+c \mathrm{e}^{v t+q u-v}+\psi_{2}+\phi_{2}\right)\right\} \\
& +\mathrm{e}^{v t+q u-v}\left(q H_{1}-H_{2}\right)-\frac{n \alpha^{2}\left|\nabla \phi_{2}\right|^{2}}{4 \beta_{2}\left(\alpha-\beta_{2}\right)\left(\psi_{2}+\phi_{2}\right)} \\
& +\mathrm{e}^{v t+q u-v}\left(c v+\psi_{2}+\phi_{2}-q\left(\psi_{1}+\phi_{1}\right)\right)+\partial_{t} \psi_{2} \\
& -\Delta \phi_{2}+\frac{2}{n \alpha^{2}}\left(\alpha-\beta_{2}\right)\left(\psi_{2}+\phi_{2}\right)^{2}
\end{aligned}
$$

This completes the proof of Lemma 2.

Next, we need to compute specific $\psi_{i}(t)$ and $\phi_{i}(x)$ that guarantee $h_{i 2}>0$ for the maximum principle to be applicable where $i=1,2$.

Lemma 3. Assume $k_{1}-p k_{2} \geq 0, k_{2}-q k_{1} \geq 0, l_{1}-p l_{2} \geq 0$, $l_{2}-q l_{1} \geq 0$ and

$$
\begin{aligned}
& k_{i} \geq \frac{n \alpha^{2}}{2\left(\alpha-\beta_{i}\right)}>0, \\
& l_{i} \geq \frac{n \alpha^{2}}{2\left(\alpha-\beta_{i}\right)}\left(6+\frac{n \alpha^{2}}{\beta_{i}\left(\alpha-\beta_{i}\right)}\right), \quad i=1,2 .
\end{aligned}
$$


If $\psi_{i}(t)=k_{i} / t, \phi_{i}(x)=\sum_{m=1}^{n}\left(l_{i} /\left(x_{m}-a_{m}\right)^{2}+l_{i} /\left(b_{m}-x_{m}\right)^{2}\right)$, $i=1,2$ for $x \in \Omega=\left[a_{1}, b_{1}\right] \times\left[a_{2}, b_{2}\right] \times \cdots\left[a_{n}, b_{n}\right]$, then for some $x_{0} \in \Omega,\left.h_{12}\right|_{x=x_{0}}>0$ and $\left.h_{22}\right|_{x=x_{0}}>0$.

Proof. From $k_{1}-p k_{2} \geq 0, k_{2}-q k_{1} \geq 0$ and $l_{1}-p l_{2} \geq 0$, $l_{2}-q l_{1} \geq 0$, we get $c \mu+\psi_{1}+\phi_{1}-p\left(\psi_{2}+\phi_{2}\right) \geq 0$ and $c \nu+\psi_{2}+$ $\phi_{2}-q\left(\psi_{1}+\phi_{1}\right) \geq 0$.

Now applying Lemma 2 yields

$$
\begin{gathered}
h_{12} \geq \partial_{t} \psi_{1}-\Delta \phi_{1}+\frac{2}{n \alpha^{2}}\left(\alpha-\beta_{1}\right)\left(\psi_{1}+\phi_{1}\right)^{2} \\
-\frac{n \alpha^{2}\left|\nabla \phi_{1}\right|^{2}}{4 \beta_{1}\left(\alpha-\beta_{1}\right)\left(\psi_{1}+\phi_{1}\right)}, \\
h_{22} \geq \partial_{t} \psi_{2}-\Delta \phi_{2}+\frac{2}{n \alpha^{2}}\left(\alpha-\beta_{2}\right)\left(\psi_{2}+\phi_{2}\right)^{2} \\
-\frac{n \alpha^{2}\left|\nabla \phi_{2}\right|^{2}}{4 \beta_{2}\left(\alpha-\beta_{2}\right)\left(\psi_{2}+\phi_{2}\right)} .
\end{gathered}
$$

By the definitions of $\psi_{i}$ and $\phi_{i}$, we obtain

$$
\begin{aligned}
\Delta \phi_{i} & =\sum_{m=1}^{n}\left(\frac{6 l_{i}}{\left(x_{m}-a_{m}\right)^{4}}+\frac{6 l_{i}}{\left(b_{m}-x_{m}\right)^{4}}\right), \\
\left|\nabla \phi_{i}\right|^{2} & =\sum_{m=1}^{n}\left(\frac{-2 l_{i}}{\left(x_{m}-a_{m}\right)^{3}}+\frac{2 l_{i}}{\left(b_{m}-x_{m}\right)^{3}}\right)^{2},
\end{aligned}
$$

and

$$
\frac{\left|\nabla \phi_{i}\right|^{2}}{\psi_{i}+\phi_{i}} \leq \sum_{m=1}^{n}\left(\frac{2 \sqrt{l_{i}}}{\left(x_{m}-a_{m}\right)^{2}}+\frac{2 \sqrt{l_{i}}}{\left(b_{m}-x_{m}\right)^{2}}\right)^{2} \text {. }
$$

If

$$
\begin{aligned}
& k_{i} \geq \frac{n \alpha^{2}}{2\left(\alpha-\beta_{i}\right)}>0, \\
& l_{i} \geq \frac{n \alpha^{2}}{2\left(\alpha-\beta_{i}\right)}\left(6+\frac{n \alpha^{2}}{\beta_{i}\left(\alpha-\beta_{i}\right)}\right), \quad i=1,2,
\end{aligned}
$$

then $h_{12}>0$ and $h_{22}>0$.

This proves Lemma 3.

Proof of Theorem 1. Choose

$$
\begin{aligned}
\psi_{i}(t) & =\frac{k_{i}}{t} \\
\phi_{i}(x) & =\sum_{m=1}^{n}\left(\frac{l_{i}}{\left(x_{m}-a_{m}\right)^{2}}+\frac{l_{i}}{\left(b_{m}-x_{m}\right)^{2}}\right),
\end{aligned}
$$

$i=1,2$ for $x \in \Omega=\left[a_{1}, b_{1}\right] \times\left[a_{2}, b_{2}\right] \times \cdots\left[a_{n}, b_{n}\right]$. Note that

$$
\begin{aligned}
\lim _{t \rightarrow 0} H_{i}=\infty=\lim _{x_{m} \longrightarrow a_{m}} H_{i}=\lim _{x_{m} \longrightarrow b_{m}} H_{i}, & \\
& \quad m=1,2, \cdots, n, i=1,2 .
\end{aligned}
$$

Assume that there exists a first time $t_{0}$ and point $x_{0} \in \Omega$ where $H_{1}\left(x_{0}, t_{0}\right)=0$ and $H_{2}\left(x_{0}, t_{0}\right)>0$. At $\left(x_{0}, t_{0}\right)$; we have

$$
\begin{gathered}
\nabla H_{1}=0, \\
\Delta H_{1} \geq 0, \\
\partial_{t} H_{1} \leq 0 .
\end{gathered}
$$

Lemma 3 implies that

$$
\begin{gathered}
\partial_{t} H_{1}-\Delta H_{1}-2 \nabla H_{1} \cdot \nabla u-h_{11} H_{1} \\
-\mathrm{e}^{\mu t+p v-u}\left(p H_{2}-H_{1}\right) \geq h_{12}>0
\end{gathered}
$$

This is a contradiction. Assume that there exist a first time $t_{0}$ and point $x_{0} \in \Omega$ where $H_{2}\left(x_{0}, t_{0}\right)=0$ and $H_{1}\left(x_{0}, t_{0}\right)>0$. At $\left(x_{0}, t_{0}\right)$, we have

$$
\begin{gathered}
\nabla H_{2}=0, \\
\Delta H_{2} \geq 0, \\
\partial_{t} H_{2} \leq 0 .
\end{gathered}
$$

Lemma 3 implies that

$$
\begin{aligned}
\partial_{t} H_{2} & -\Delta H_{2}-2 \nabla H_{2} \cdot \nabla v-h_{21} H_{2} \\
& -e^{v t+q u-v}\left(q H_{1}-H_{2}\right) \geq h_{22},
\end{aligned}
$$

This is a contradiction. Furthermore, $H_{1}\left(x_{0}, t_{0}\right)=0$ and $H_{2}\left(x_{0}, t_{0}\right)=0$ cause the same contradiction as $H_{1}\left(x_{0}, t_{0}\right)=0$ and $H_{2}\left(x_{0}, t_{0}\right)>0$. Thus $H_{1}(x, t)>0$ and $H_{2}(x, t)>0$ for all $x, t>0$.

Taking $\Omega \longrightarrow \mathbb{R}^{n}$ which obtains $\phi_{i} \longrightarrow 0$ then gives the desired result.

\section{Applications}

In this section, we shall give an application of Theorem 1 . We integrate along space-time to derive a classical Harnack inequality.

3.1. Classical Harnack Inequality. In this subsection, we integrate our differential Harnack inequality of Theorem 1 along space-time to derive a classical Harnack inequality.

Proposition 4. Let $(f(x, t), g(x, t))$ be positive classical solutions to (1) and $(u(x, t), v(x, t)):=(\log f, \log g)$. Suppose that $x_{1}, x_{2} \in \mathbb{R}^{n}$ and $t_{2}>t_{1}>0$. Assume further that $\alpha \geq$ $2 \max \left\{\beta_{1}, \beta_{2}\right\}, \alpha \geq c$ and $k_{i}=n \alpha^{2} / 2\left(\alpha-\beta_{i}\right), i=1,2$. Then we have

$$
\begin{aligned}
& f\left(x_{1}, t_{1}\right) \leq f\left(x_{2}, t_{2}\right)\left(\frac{t_{2}}{t_{1}}\right)^{n} \exp \left(\frac{\left|x_{2}-x_{1}\right|^{2}}{2\left(t_{2}-t_{1}\right)}\right), \\
& g\left(x_{1}, t_{1}\right) \leq g\left(x_{2}, t_{2}\right)\left(\frac{t_{2}}{t_{1}}\right)^{n} \exp \left(\frac{\left|x_{2}-x_{1}\right|^{2}}{2\left(t_{2}-t_{1}\right)}\right) .
\end{aligned}
$$

Proof. Define the one-variable functions $w_{i}:\left[t_{1}, t_{2}\right] \rightarrow \mathbb{R}$ as

$$
w_{1}(t):=u(\gamma(t), t),
$$




$$
w_{2}(t):=v(\gamma(t), t)
$$

for any $C^{1}$ path $\gamma:\left[t_{1}, t_{2}\right] \longrightarrow \mathbb{R}^{n}$ such that $\gamma\left(t_{1}\right)=x_{1}, \gamma\left(t_{2}\right)=$ $x_{2}$.

Applying Theorem 1, we have

$$
\Delta u \geq-\alpha^{-1}\left(\beta_{1}|\nabla u|^{2}+c \mathrm{e}^{\mu t+p v-u}+\frac{k_{1}}{t}\right) .
$$

It yields that

$$
\begin{aligned}
\frac{d}{d t} w_{1}(t)= & u_{t}+\nabla u \cdot \frac{d \gamma}{d t} \\
= & \Delta u+|\nabla u|^{2}+\mathrm{e}^{\mu t+p v-u}+\nabla u \cdot \frac{d \gamma}{d t} \\
\geq & -\alpha^{-1}\left(\beta_{1}|\nabla u|^{2}+c \mathrm{e}^{\mu t+p v-u}+\frac{k_{1}}{t}\right)+|\nabla u|^{2} \\
& +\mathrm{e}^{\mu t+p v-u}+\nabla u \cdot \frac{d \gamma}{d t} \\
\geq & \left(\frac{1}{2}-\frac{\beta_{1}}{\alpha}\right)|\nabla u|^{2}+\left(1-\frac{c}{\alpha}\right) \mathrm{e}^{\mu t+p v-u}-\frac{k_{1}}{\alpha t} \\
& -\frac{1}{2}\left|\frac{d \gamma}{d t}\right|^{2} \geq-\frac{1}{2}\left|\frac{d \gamma}{d t}\right|^{2}-\frac{n}{t},
\end{aligned}
$$

where $\alpha \geq 0, \alpha \geq 2 \beta_{1}, \alpha \geq c$ and $k_{1}=n \alpha^{2} / 2\left(\alpha-\beta_{1}\right)$. Similarly,

$$
\frac{d}{d t} w_{2}(t) \geq-\frac{1}{2}\left|\frac{d \gamma}{d t}\right|^{2}-\frac{n}{t}
$$

for $\alpha \geq 0, \alpha \geq 2 \beta_{2}, \alpha \geq c$ and $k_{2}=n \alpha^{2} / 2\left(\alpha-\beta_{2}\right)$.

By

$$
\begin{aligned}
& \int_{t_{1}}^{t_{2}}\left|\frac{d \gamma}{d t}\right|^{2} d t \geq \frac{\left|x_{2}-x_{1}\right|^{2}}{t_{2}-t_{1}} \\
& \int_{t_{1}}^{t_{2}}-\frac{d}{d t} w_{i}(t) \leq \inf _{\gamma(t)} \int_{t_{1}}^{t_{2}}\left(\frac{1}{2}\left|\frac{d \gamma}{d t}\right|^{2}+\frac{n}{t}\right) d t, \quad i=1,2,
\end{aligned}
$$

applying $(u, v)=(\log f, \log g)$ gives Proposition 4 .

\section{Data Availability}

No data were used to support this study.

\section{Conflicts of Interest}

The author declares that there are no conflicts of interest regarding the publication of this paper.

\section{Acknowledgments}

This research is supported by the National Natural Science Foundation of China (No. 11801306).

\section{References}

[1] P. Li and S.-T. Yau, "On the parabolic kernel of the Schrödinger operator," Acta Mathematica, vol. 156, no. 1, pp. 153-201, 1986.

[2] R. S. Hamilton, "Li-Yau estimates and their Harnack inequalities," in Geometry and Analysis, vol. 17 of Advanced Lectures in Mathematics (ALM), pp. 329-362, International Press, Massachusetts, Mass, USA, 2011.

[3] H. Wu and L. Min, "Differential Harnack estimate for a semilinear parabolic equation on hyperbolic space," Applied Mathematics Letters, vol. 50, pp. 69-77, 2015.

[4] X. S. Du and A. M. Mao, "Existence and multiplicity of nontrivial solutions for a class of semilinear fractional Schrödinger equations," Journal of Function Spaces, vol. 2017, Article ID 3793872, 7 pages, 2017.

[5] Q. Feng and F. Meng, "Traveling wave solutions for fractional partial differential equations arising in mathematical physics by an improved fractional Jacobi elliptic equation method," Mathematical Methods in the Applied Sciences, vol. 40, no. 10, pp. 3676-3686, 2017.

[6] F. S. Li, "Global existence and uniqueness of weak solution for nonlinear viscoelastic full Marguerre-von Karman shallow shell equations," Acta Mathematica Sinica, vol. 25, no. 12, pp. 2133 2156, 2009.

[7] F. Li, "Backward solutions to neumann and dirichlet problems of heat-conduction equation," Applied Mathematics and Computation, vol. 210, no. 1, pp. 211-214, 2009.

[8] F. Li, Z. Zhao, and Y. Chen, "Global existence uniqueness and decay estimates for nonlinear viscoelastic wave equation with boundary dissipation," Nonlinear Analysis: Real World Applications, vol. 12, no. 3, pp. 1759-1773, 2011.

[9] F. S. Li and J. L. Li, "Global existence and blow-up phenomena for nonlinear divergence form parabolic equations with inhomogeneous Neumann boundary conditions," Journal of Mathematical Analysis and Applications, vol. 385, no. 2, pp. 1005-1014, 2012.

[10] F. Li and J. Li, "Global existence and blow-up phenomena for p-Laplacian heat equation with inhomogeneous Neumann boundary conditions," Boundary Value Problems, no. 219, pp. 114, 2014.

[11] H. Liu, "The homogeneous polynomial solutions for the Grushin operator," Acta Mathematica Scientia B, vol. 38, no. 1, pp. 237-247, 2018.

[12] L. Ren and J. Xin, "Almost global existence for the Neumann problem of quasilinear wave equations outside star-shaped domains in 3D," Electronic Journal of Differential Equations, vol. 312, pp. 1-22, 2017.

[13] M. Shao and A. Mao, "Multiplicity of solutions to SchrödingerPoisson system with concave-convex nonlinearities," Applied Mathematics Letters, vol. 83, pp. 212-218, 2018.

[14] H. Wu and X. Yang, "Global existence and finite time blowup for a parabolic system on hyperbolic space," Journal of Mathematical Physics, vol. 59, pp. 1-11, 2018.

[15] Y. Xu and H. Zhang, "Multiple positive solutions of a mpoint boundary value problem for 2nth-order singular integrodifferential equations in Banach spaces," Nonlinear Analysis: Theory, Methods \& Applications, vol. 70, no. 9, pp. 3243-3253, 2009.

[16] Y. Xu and H. Zhang, "Multiple positive solutions of a boundary value problem for a class of $2 \mathrm{nth}$-order m-point singular integro-differential equations in Banach spaces," Applied Mathematics and Computation, vol. 214, no. 2, pp. 607-617, 2009. 
[17] X. Zheng, Y. Shang, and H. Di, "The time-periodic solutions to the modified Zakharov equations with a quantum correction," Mediterranean Journal of Mathematics, vol. 14, pp. 1-17, 2017.

[18] F. Li and Q. Gao, "Blow-up of solution for a nonlinear Petrovsky type equation with memory," Applied Mathematics and Computation, vol. 274, pp. 383-392, 2016.

[19] H. Wu, "Blow-up and nonexistence of solutions of some semilinear degenerate parabolic equations," Boundary Value Problems, vol. 157, pp. 1-12, 2015.

[20] H. Wu, "A Fujita-type result for a semilinear equation in hyperbolic space," Journal of the Australian Mathematical Society, vol. 103, no. 3, pp. 420-429, 2017.

[21] $\mathrm{H}$. Wu, "Liouville-type theorem for a nonlinear degenerate parabolic system of inequalities," Mathematical Notes, vol. 103, no. 1-2, pp. 155-163, 2018.

[22] M. Zhu and Y. Wang, "Blow-up of solutions to the rotation b-family system modeling equatorial water waves," Electronic Journal of Differential Equations, vol. 78, pp. 1-23, 2018.

[23] Y.-H. Feng and C.-M. Liu, "Stability of steady-state solutions to navier-stokes-poisson systems," Journal of Mathematical Analysis and Applications, vol. 462, no. 2, pp. 1679-1694, 2018.

[24] F. Li and Y. Bai, "Uniform rates of decay for nonlinear viscoelastic Marguerre-von Karman shallow shell system," Journal of Mathematical Analysis and Applications, vol. 351, no. 2, pp. 522-535, 2009.

[25] F. Li, "Limit behavior of the solution to nonlinear viscoelastic Marguerre -von Karman shallow shells system," Journal of Differential Equations, vol. 249, no. 6, pp. 1241-1257, 2010.

[26] F. Li and Y. Bao, "Uniform stability of the solution for a memorytype elasticity system with nonhomogeneous boundary control condition," Journal of Dynamical and Control Systems, vol. 23, no. 2, pp. 301-315, 2017.

[27] F. S. Li and G. W. Du, "General energy decay for a degenerate viscoelastic petrovsky-type plate equation with boundary feedback," Journal of Applied Analysis and Computation, vol. 8, no. 1, pp. 390-401, 2018.

[28] H. Liu, F. Liu, and H. Wu, "The unique continuation property of p-harmonic functions on the Heisenberg group," Bulletin of the Australian Mathematical Society, vol. 99, no. 2, pp. 219-230, 2019. 


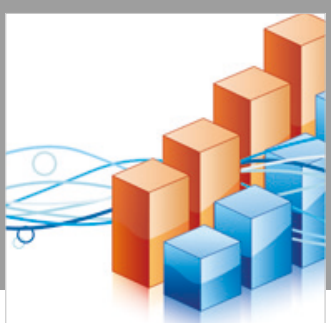

Advances in

Operations Research

\section{-n-m}
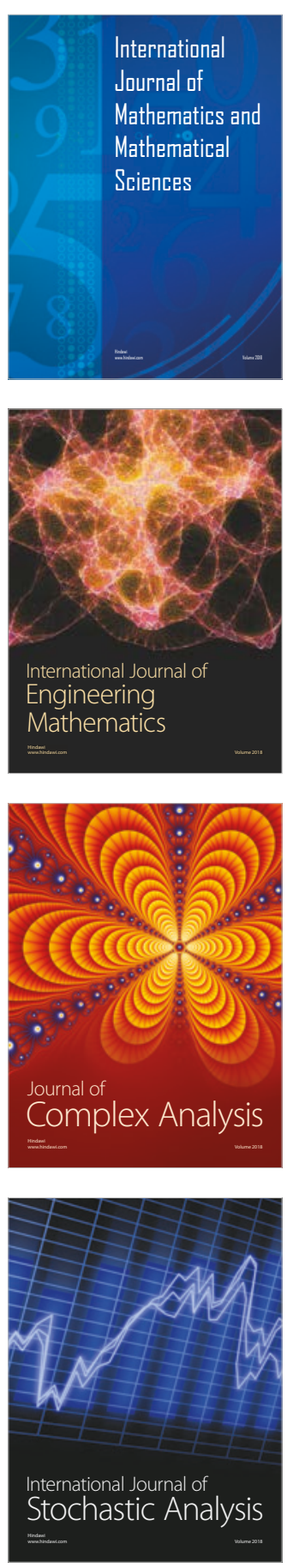
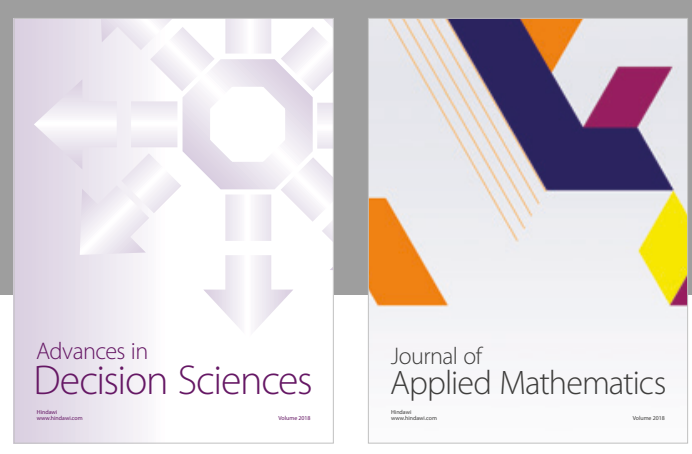

Journal of

Applied Mathematics
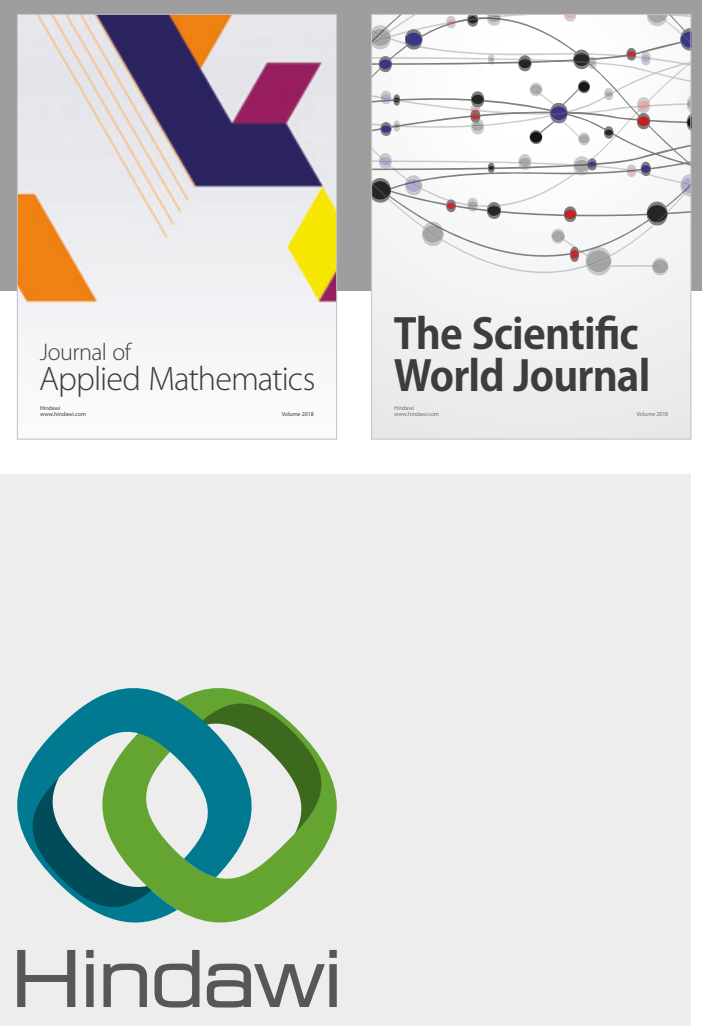

Submit your manuscripts at

www.hindawi.com

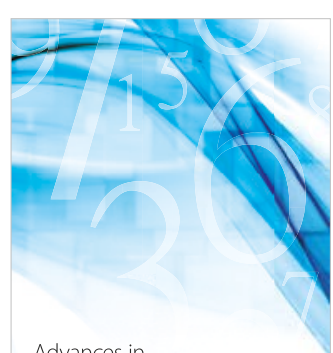

Advances in
Numerical Analysis
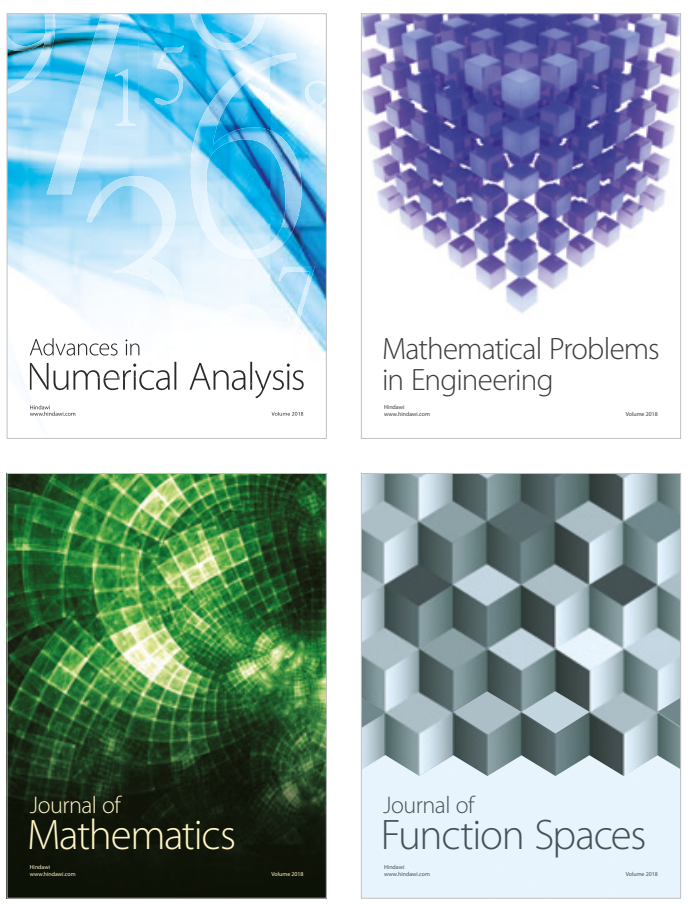

Mathematical Problems in Engineering

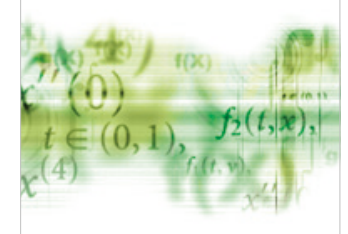

International Journal of

Differential Equations

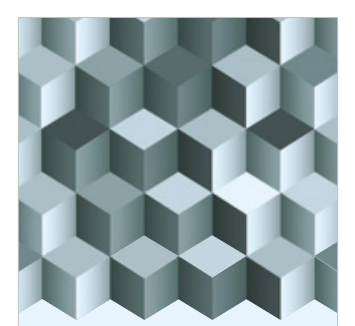

Journal of

Function Spaces

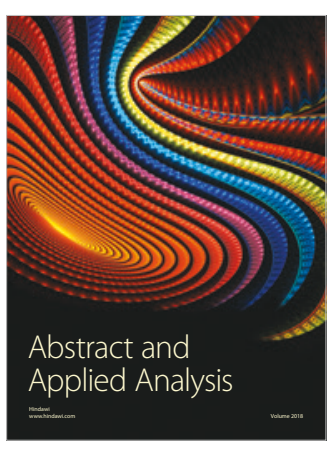

The Scientific

World Journal

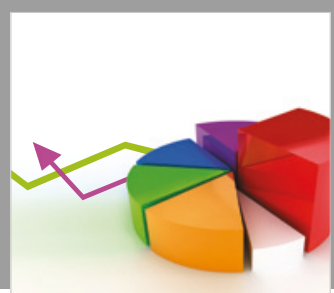

Journal of

Probability and Statistics
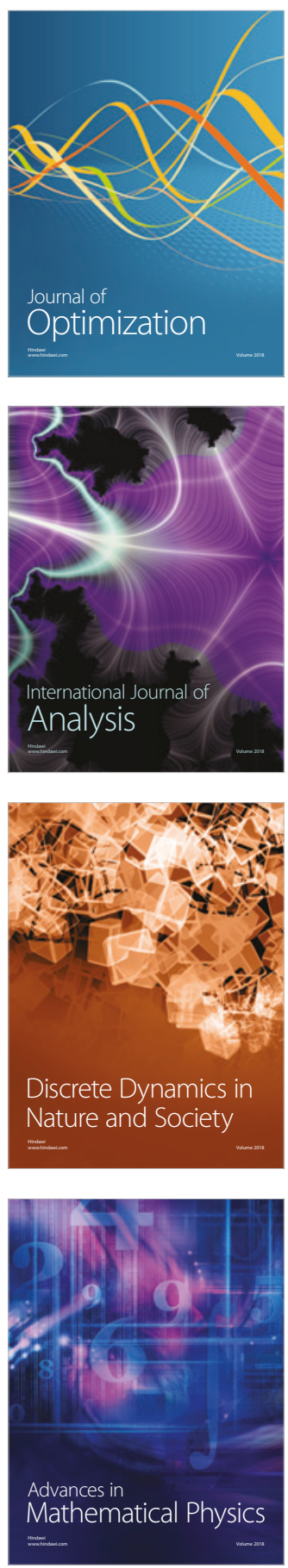